\title{
Ernst von Leyden und die Institutionalisierung der Krebsforschung zwischen 1896 und 1911
}

Thorsten Kohl

Ernst von Leyden and the Institutionalization of Cancer Research Between 1896 and 1911

The institutionalization of cancer research in the German context was initiated by formation of the "Comite für Krebsforschung" (Committee for Cancer Research) in February 1900. One of the main actors in this connection was Ernst von Leyden (1832-1910), physician, clinician and head of the First Clinic of the Berliner Charité. This article investigates the essential conditions for the process of institutionalization and its further development in time. For this purpose, the concept of "resources" and a multi-level model of the public are applied. It becomes apparent that bacteriology as well as statistics and tuberculosis research were not deployed as scientific resources, but rather as instruments of science policy. The period of investigation comprises the later publications of Leyden, concerning the etiology of cancer, the foundation of the "Comité" and its expansion right up to the participation of its members in exhibitions, namely the "Internationale Hygiene-Ausstellung" (International Hygiene Exhibition) in Dresden 1911.

Keywords: Institutionalization, history of cancer research, physician Ernst von Leyden, resource model, stage model of public

Schlüsselwörter: Institutionalisierung, Geschichte der Krebsforschung, Mediziner Ernst von Leyden, Ressourcenmodell, Stufenmodell der Öffentlichkeit

Die Institutionalisierung der Krebsforschung im deutschsprachigen Raum begann um 1900 nicht unbescheiden. Die Gründung einer eigenen Gesellschaft, einer Fachzeitschrift sowie dedizierter medizinischer Krebsforschungsinstitute mit unterschiedlichen Arbeitsschwerpunkten bildeten den Rahmen. Die Ausrichtung erster onkologischer Fachkongresse bis hin zur Bildung einer Gruppe „Krebs“ innerhalb der wissenschaftlichen Abteilung der Internationalen Hygiene-Ausstellung 1911 in Dresden erweiterten in der Folge zunehmend den Adressatenkreis über die engeren Fachdisziplinen hinaus. Federführend war zunächst eine kleine Gruppe von Spezialisten mit Erfahrungen in der Bakteriologie, der Statistik und der Tuberkulosebekämpfung, die 
als Professoren an den medizinischen Fakultäten verschiedener Universitäten oder im Gesundheitsbereich des Verwaltungsapparats tätig waren. Einer der Hauptakteure war der Mediziner Ernst von Leyden (1832-1910), der in der Zeit von 1885 bis 1907 der I. Medizinischen Klinik der Berliner Charité vorstand.

In der Medizingeschichte wurden die Institutionalisierung der Krebsforschung und der Beitrag von Leyden bereits mehrfach untersucht. Zuletzt thematisierten Isabel Atzl und Roland Helms in ihrer Geschichte der Deutschen Krebsgesellschaft (Atzl und Helms 2012) die Gründung des „Comités für Krebsforschung“, mit der die Geschichte der Deutschen Krebsgesellschaft und der organisierten Krebsforschung beginnt, aus institutionsgeschichtlicher Perspektive. Zuvor hatte Peter Schneck (2000) die Vorgeschichte der Krebsforschung im deutschsprachigen Kontext beleuchtet. Demnach ist in der „Aufforderung zu einer vorläufigen Erhebung über das Vorkommen von Krebserkrankungen in Preußen“, die 1899 durch den preußischen Minister der geistlichen, Unterrichts- und Medizinalangelegenheiten erging, der erste Hinweis auf die Anfänge der organisierten Krebsforschung zu erkennen (ebd.: 23). 1992 hatte Andreas Ebert zudem ein „95 Seiten starkes Kontobuch mit dem Etikett ,Comité für Krebsforschung. Protokollbuch I vom 18. Februar 1900 - 2. November 1907“" vorgestellt, sich dabei aber auf eine knappe Beschreibung und Einordnung dieses Archivfundes beschränkt.

Der vorliegende Beitrag knüpft an die genannten Arbeiten an, erweitert diese aber durch den analytischen Rahmen und fragt nach den wissenschaftspolitischen Rahmenbedingungen der Institutionalisierung der Krebsforschung. Dabei werden die Strategien der Institutionalisierung verfolgt, die über die Gründung des Komitees hinausgehen. Ausgehend vom Forschungsstand wird Ernst von Leyden als einer der zentralen Akteure in diesem Prozess angesehen. Vor allem wird gezeigt, wie die Krebsforschung mittels Statistik, Tuberkuloseforschung und Bakteriologie die angestrebten Ziele umsetzte.

Zur Interpretation dieser Entwicklung wird das Ressourcenkonzept des Historikers Mitchell G. Ash herangezogen (Ash 2001, 2007). Ash legt in seinem Beitrag "Wissenschaft und Politik als Ressourcen füreinander" eine Redeweise nahe, die es erlaubt, das Verhältnis von Wissenschaft und Politik moderner Gesellschaften kritisch zu thematisieren. Es geht ihm dabei sowohl um die sich wandelnden Vernetzungen von Wissenschaft und Politik als auch um die Wandlungen dessen, was als Politik und was als Wissenschaft anzusehen ist. Aus seiner Sicht sind Wissenschaftswandel und -kontinuität als Fortsetzung und Umgestaltung von Ressourcenensembles beschreibbar.

Unter Ressourcen werden in diesem Zusammenhang nicht nur finanzielle Ressourcen verstanden, sondern sie können auch „kognitiver, apparativer, personeller, institutioneller oder rhetorischer Art" sein. Nach Ash sind die 
Ressourcenensembles prinzipiell politisch multivalent und gegenseitig mobilisierbar.

Im Folgenden soll eine Ressource im Prozess der Institutionalisierung der Krebsforschung als „wissenschaftliche Ressource“ bezeichnet werden, wenn deren Mobilisierung primär dem Erkenntnisgewinn in Bezug auf die Krebsätiologie, die Diagnostik oder die Therapie diente. Demgegenüber stützte eine Mobilisierung rhetorischer und wissenschaftspolitischer Ressourcen zunächst den Institutionalisierungsprozess selbst. Wissenschaftspolitische Ressourcen unterliegen hierbei nicht unbedingt Kriterien wie Verifizierbarkeit oder wissenschaftlichem common sense. Somit ist das entscheidende Kriterium für die Einordnung einer Ressource der Zweck bzw. das Ziel ihrer Mobilisierung. Im Rahmen des vorliegenden Aufsatzes werden insbesondere Personen und wissenschaftliche Ressourcen (Bakteriologie, Statistik und Tuberkuloseforschung) als die wesentlichen Ressourcen begriffen, die den Institutionalisierungsprozess stützten und im Verlauf des Prozesses eine mehrfache Rekonfiguration erfuhren.

Darüber hinaus soll verdeutlicht werden, dass der zeitliche Verlauf der Institutionalisierung mit einer zunehmenden, schritt- oder stufenweisen Einbindung verschiedener Teile der Öffentlichkeit einherging. Eine angemessene Beschreibung dieser Entwicklung ermöglicht das „Stufenmodell der Öffentlichkeit" nach Arne Schirrmacher. Schirrmacher führt die von Ludwig Fleck vorgenommene Dreiteilung der Sphäre der Wissenschaft mit dem von Gabriel Almond vorgeschlagenen Modell einer ebenfalls dreiteiligen Differenzierung der Öffentlichkeit zusammen (Schirrmacher 2008: 84; Nikolow et al. 2007: 30). Er erhält so ein sechs Ebenen umfassendes „Stufenmodell der Öffentlichkeit", das ausgehend von der Fachwissenschaft (F), über die Fachkreise außerhalb des engeren Forschungsgebietes $\left(\mathrm{F}_{2}\right)$ und der Fachöffentlichkeit (FÖ), die gebildete und interessierte $\left(\mathrm{O}_{3}\right)$, die gelegentlich interessierte $\left(\ddot{O}_{2}\right)$ und schließlich die breite Öffentlichkeit $\left(\ddot{O}_{1}\right)$ integriert (ebd.: 86). Dieses Modell dient dazu, die „Pluralisierung von Vermittlungsdiskursen und Grenzziehungsarbeiten“ zwischen Wissenschaft und Öffentlichkeit zu untersuchen. Hierbei betont Schirrmacher, unter Bezugnahme auf Ash, auch die komplexen Beziehungen zwischen Wissenschaften und Öffentlichkeiten im 20. Jahrhundert, die als wechselseitige Ressourcenbeziehungen beschrieben werden können (ebd.: 89). Schirrmachers Modell ermöglicht es, die Dynamik der Institutionalisierung, ausgehend von einer Gruppe Fachwissenschaftler um Ernst von Leyden bis hin zur Adressierung eines breiten, öffentlichen Publikums auf der Hygiene-Ausstellung 1911, zu erfassen und dabei die mehrfache Rekonfiguration der Ressourcenensembles während des Verlaufs abzubilden. Für die Beschreibung dieses Kontexts des Institutionalisierungsprozesses, unter anderem mit Blick auf Tuberkuloseforschung, Bakteriologie und Ausstellungsgeschichte, wird weitere einschlägige 
Forschungsliteratur einbezogen (Condrau 2000; Berger 2009; Gradmann 2010; Brecht und Nikolow 2000).

Der Beitrag geht von der These aus, dass Leyden in den 1890er Jahren eine geschickte Mobilisierung unterschiedlichster Ressourcen gelang, die schließlich die Konstituierung des „Comités für Krebsforschung" ermöglichte. Um die Ziele des Komitees zu erreichen, mussten weitere Öffentlichkeiten eingebunden werden, deren Adressierung einen Wandel von Ressourcenensembles voraussetzte. Im weiteren Verlauf des Beitrages wird der Frage nach der stufenweisen Einbindung weiterer Öffentlichkeiten sowie dem Wandel der Ressourcenensembles nachgegangen.

\section{Motive: Volks-Heilstätten und Krebs-Parasiten}

In seinen Lebenserinnerungen widmete Ernst von Leyden der Krebsforschung ein eigenes Kapitel. Einleitend schrieb er: „Lange schon hatte mich das Bestreben erfüllt, nachdem die Tuberkulosefrage einen so erfreulichen Abschluß gefunden, gegen eine andere Volkskrankheit - die Krebskrankheit (Karzinom) - [...] den Kampf aufzunehmen." (Lohde-Boetticher 1910: 249). Leydens Einschätzung in Bezug auf den Abschluss der Tuberkulosefrage wirkt irritierend und wirft die Frage auf, worin er den „erfreulichen Abschluß“ der Tuberkulosefrage zu erkennen glaubte. Immerhin wies die Statistik die Tuberkulose um 1900 noch als die häufigste Todesursache aus, Krebs folgte in einem deutlichen Abstand. Ein wirksamer Tuberkuloseimpfstoff war noch nicht verfügbar. Dennoch bestand für Leyden, im Gegensatz zu Robert Koch (1843-1910), die Lösung der Tuberkulosefrage nicht ausschließlich in der Verfügbarkeit eines wirksamen Mittels gegen das infektiöse Agens. Seinem Ziel und klinischem Selbstverständnis entsprach die Errichtung von Sanatorien und Heilstätten zur Behandlung der Lungenschwindsucht (Leyden 1894). In einem Vortrag über die Lungentuberkulose legte er im September 1894 seine Position folgendermaßen dar: „Die hygienisch-diätische Behandlung verbunden mit der Anstaltsbehandlung ist demnach auch heute als die beste Heilmethode anerkannt." (Ebd.: 6)

Flurin Condrau verweist in diesem Zusammenhang darauf, dass ein spezifisches Heilmittel gegen die Tuberkulose jede Art von Heilstätte sofort überflüssig gemacht hätte (Condrau 2000: 121). So kam es nach der Ankündigung des Tuberkulins durch Koch 1890 sofort „zu einem Stillstand der Diskussion um die Heilstätten." (Ebd.) Kochs optimistische Darstellung weckte weitreichende Hoffnungen und endete spätestens 1891 ernüchternd. Die Wirksamkeit stellte sich als fraglich heraus, die Anwendung wurde von schweren Nebenwirkungen bis hin zu Todesfällen begleitet, Koch geriet in die Kritik. Auch Leyden griff ihn an und kritisierte sein Verhalten im Umgang mit dem Tuberkulin heftig (Gradmann 2010: 210). 
Der „Tuberkulinrausch von 1890“ (Elkeles 1990; Schadewaldt 1982) geriet für den Kliniker Leyden zu einem Alptraum, da seine Anstrengungen zur Errichtung von Heilstätten Gefahr liefen zu scheitern. Nach Abflauen der Tuberkulin-Begeisterung führte er 1894 in einem Vortrag auf dem Internationalen Hygiene-Kongress in Budapest nahezu erleichtert aus,

dass [d]ie Anerkennung dieser Methode nur eine kurze Unterbrechung erfahren [habe], als nach der Entdeckung des Tuberculins die Hoffnung auf ein specifisches Heilmittel in einem ganz ungewöhnlichen Grade belebt wurde. Heute erkennen wir wieder die Anstaltsbehandlung als die beste an und hegen die berechtigte Meinung, dass sie für lange Zeit die beste bleiben wird, selbst dann, wenn die Hoffnung auf die Entdeckung specifischer Heilmittel sich mehr erfüllen sollte, als es bisher den Anschein hat. (Leyden 1894: 6)

In seiner 14 Jahre später erschienenen Biografie wies Leyden erneut darauf hin, dass man nach dem Verklingen des „Tuberkulinrausches“ begreiflicherweise zu den älteren Heilmethoden zurückkehrte: den Luft- und diätetischen Behandlungen (Lohde-Boetticher 1910: 181).

Auch wenn die Heilstätten der Kritik ausgesetzt waren, weil die Heilerfolge keinesfalls überzeugten, verstand sich Leyden in dieser Situation vor allem als Kliniker, eine Position die im Folgenden deutlich zum Ausdruck kommt: „Als höchste Leistung der ärztlichen Kunst möchte ich es bezeichnen, in den schwierigen und verzweifelten Fällen aus den Schätzen der Wissenschaft und der Erfahrung die richtigen Mittel und Wege zu suchen und finden, die zur Heilung führen." (Ebd.: 162) Das Tuberkulin fand sich für Leyden nicht unter diesen "Schätzen der Wissenschaft". Und in Bezug auf die Krebsbehandlung brachte er noch einmal zum Ausdruck, dass die Entwicklung eines Therapeutikums in absehbarer Zukunft nicht zu erwarten war.

Dennoch besaß die wissenschaftliche Bakteriologie für Leyden eine hohe Relevanz, hatte sich aber als wissenschaftliche Methode als eine Hilfswissenschaft wie andere auch den klinischen Rahmenbedingungen unterzuordnen. Er konnte auf seine eigenen bakteriologischen Arbeiten verweisen, die die Suche nach Erregern von Lungenkrankheiten und der Cholera zum Inhalt hatten und auch Versuche zur Herstellung eines Heilserums für die Pneumonie umfassten. Seine wissenschaftliche Erfahrung zeigte ihm aber deutlich, dass die Identifizierung eines Krankheitserregers nicht unmittelbar zu einem Heilmittel führen musste. Das theoretische Wissen über die Entstehung einer Krankheit und der praktische, klinische Umgang mit ihr waren aus seiner Sicht voneinander zu unterscheiden.

Leydens Motivation, sich aktiv für eine Institutionalisierung der Krebsforschung einzusetzen, leitete sich aus seinen späten experimentellen Arbeiten zur Krebsätiologie ab. Sie war somit zunächst nicht durch eine klinische Relevanz, sondern durch einen theoretischen Forschungsansatz bedingt. Dieser Sacherhalt ist auch für Leydens späteren Umgang mit der Öffentlichkeit von Bedeutung. Zwischen 1896 und 1904 veröffentlichte er eine Reihe von 
Aufsätzen, die sich mit der Frage der Krebsätiologie beschäftigten. In einem ersten, 1896 gemeinsam mit Fritz Schaudinn publizierten Artikel (Leyden und Schaudinn 1896: 952), berichteten die Autoren über zwei Ascites-Patienten, in deren Punktionsflüssigkeit ungewöhnliche Zellen nachgewiesen werden konnten, die Leyden für Protozoen hielt. Zeitgleich wurde bei beiden Patienten eine Tumorerkrankung diagnostiziert.

Fritz Schaudinn (1871-1906), zu dieser Zeit Assistent am Zoologischen Institut der Universität Berlin, nahm die mikrobiologische Charakterisierung des Mikroorganismus vor und zeigte, dass es sich um einen parasitären, amöbenähnlichen Rhizopoden handelte, der die Bezeichnung Leydenia gemmipara erhielt. Fokussierte der erste, gemeinsam von Leyden und Schaudinn publizierte Aufsatz auf die Krebsdiagnose und die mikrobiologische Charakterisierung des Mikroorganismus, ohne beide Sachverhalte in einen kausalen Zusammenhang zu bringen, begann Leyden in den anschließenden Publikationen und Vorträgen diese kausale Abhängigkeit auf- und auszubauen. Als nach dem ersten, gemeinsam publizierten Artikel Schaudinn aus der Zusammenarbeit ausschied, arbeitete sich Ludwig Feinberg in die Untersuchungsmethode ein (Leyden 1901: 4). Später nahm Feinberg zu einer Demonstration mikroskopischer Präparate in der Charité-Gesellschaft Stellung und warf Leyden vor, Präparate während seiner vorübergehenden Abwesenheit und ohne Absprache demonstriert zu haben. Auch vor inhaltlicher Kritik scheute Feinberg nicht zurück (Feinberg 1907: V). Seine Publikationen weisen ihn als den eigentlichen Experten in Bezug auf die experimentellen Arbeiten aus.

Leyden brach die Versuche mit Leydenia gemmipara spätestens 1901 ab, da Züchtungsversuche keine positiven Ergebnisse zeigten. Kultivierungsversuche waren aufgrund verschiedener experimenteller Schwierigkeiten grundsätzlich nicht einfach durchzuführen. Leyden hatte unmittelbar nach dem Scheitern dieser Versuche Plasmodiophora Brassicae als möglichen alternativen Krebsparasiten eingeführt. Dieser Mikroorganismus wurde bereits in den 1880er Jahren als Erreger der Kohlhernie, einer Geschwulstkrankheit der Kohlpflanze, nachgewiesen. Leyden stützte sich hierbei weitgehend auf Analogiebetrachtungen in Bezug auf die mikroskopischen Färbetechniken. Forderungen nach Kultivierung und Überimpfung wies er jetzt ganz zurück. Seine Position in dieser Frage verteidigte er in der Konstituierungsphase der Krebsforschung offensiv, in den späteren Jahren wurde er zurückhaltender, blieb aber 1907 in der Internationalen Wochenschrift für Wissenschaft, Kunst und Technik dabei, dass er die "parasitäre Theorie lange verteidigt" habe und auch „heute noch nicht von ihr abgekommen“ sei (Leyden 1907: 1195).

Leydens experimentelle und rhetorische Praxis steht im deutlichen Widerspruch zu seinem Anspruch auf Wissenschaftlichkeit. Warum dann seine offensive Rhetorik? Von der Erklärung der Krebserkrankung auf 
Grundlage eines infektiösen Agens ging eine starke Anziehungskraft auf die Forscher aus. Dennoch gab es anerkannte wissenschaftliche Standards, wie beispielsweise die Koch'schen Postulate, denen sich Leyden in einer Art und Weise widersetze, die es als zweifelhaft erscheinen lässt, ob die Klärung der Ätiologie der Krebserkrankung Leydens vorrangiges Ziel war. Diese Beobachtung führt $\mathrm{zu}$ der These, dass das bakteriologische Modell als eine wissenschaftspolitische Ressource mobilisiert wurde.

\section{Ressourcenmobilisierung: die Gründung des „Comités für Krebsforschung'1}

Am Anfang des von Leyden seit Langem als „für notwendig erachtete[n] Plan[s], eine systematische Erforschung des Karzinoms ins Werk zu setzen“, stand seine 1896 einsetzende Forschungs- und Publikationstätigkeit, die auf den Nachweis einer parasitären Ätiologie der Krebskrankheit zielte. Dringlich schien das „Krebsproblem“ durch die statistische Häufigkeit der Krankheit und der krebsbedingten Todesfälle, auch wenn die statistischen Ergebnisse in wissenschaftlichen Kreisen durchaus kontrovers interpretiert wurden. So wurden schon frühzeitig demographische Entwicklungen oder die erweiterten diagnostischen Möglichkeiten für einen Anstieg der Zahlen verantwortlich gemacht, die Zahlen waren aber trotz allem - gerade im Gegensatz zur Ätiologiedebatte - konsensfähig(er).

Die wissenschaftspolitischen Handlungsspielräume wurden demgegenüber durch die Bakteriologie festgelegt, nach Berger die „staatstragende und vom Staat getragene Wissenschaft" (Berger 2009: 170). Leyden verstand es um 1900 argumentativ an die Tuberkuloseforschung anzuknüpfen und eine Institutionalisierung mit der Hoffnung auf zeitnahe Forschungsergebnisse zu legitimieren. Seine eigenen Publikationen unterstützten diese Argumentation wesentlich, auch wenn ihm offensichtlich klar war, was zeitnah im klinischen Bereich zum Vorteil der Patienten zu erreichen war. Eine Therapie auf Grundlage bakteriologischer Forschung zählte für ihn nicht dazu. Sein Ziel war die Einrichtung von Heilstätten zu ermöglichen. Somit hatte die Bezugnahme auf die Tuberkuloseforschung zwei Aufgaben zu erfüllen: Eine direkte, in Form einer Handlungsanleitung für die geplante Institutionalisierung, und indirekt, als zweiten Schritt im Sinne Leydens, das Ziel der Einrichtung von Heilstätten für Krebskranke zu vermitteln. Wie die Statistik wurde auch die Ätiologie des Krebses seinerzeit in den wissenschaftlichen Kreisen äußerst kontrovers diskutiert (Lüdtke 2002, 2007).

Leyden erkannte das Problem. Als es am 18. Februar 1900 auf Initiative und unter dem Vorsitz von Ernst von Leyden zur Gründung des „Comités für Krebsforschung" kam, mussten die Teilnehmer der konstituierenden Sitzung 
somit die Statistik, Bakteriologie und Tuberkuloseforschung entsprechend repräsentieren. Die von den Gründungsmitgliedern vertretenen Institutionen machten die konstituierende Sitzung auf der institutionellen Ebene scheinbar $\mathrm{zu}$ einem interdisziplinären Akt, auf professioneller Ebene jedoch ist eine interdisziplinäre Konfiguration weniger gegeben. Die folgende Tabelle zeigt die Einordnung der Teilnehmer der konstituierenden Sitzung in Bezug auf ihre institutionelle und professionelle Zugehörigkeit (Tabelle 1):

Hinsichtlich der akademischen Laufbahn der Mitglieder ist die konstituierende Sitzung als eine überwiegend fachwissenschaftliche Veranstaltung zu begreifen. Fast alle Teilnehmer hatten eine medizinische Ausbildung durchlaufen, mit Ausnahme des Juristen und Verwaltungsbeamten Dr. jur. Richard Freund. Eine nähere Betrachtung zeigt darüber hinaus, dass alle Akteure aus dem Umfeld der Hygiene, der Bakteriologie oder der Tuberkulosebekämpfung kamen. Die Vertreter der statistischen Ämter lieferten die Daten, auf die sich Leyden bei seiner Argumentation stützte. Zentral war auch die Doppelfunktion der Akteure.

Als Beispiele für die gezielte Mobilisierung personeller Ressourcen seien Martin Kirchner und Albert Guttstadt genannt: Kirchner fiel als Mediziner und Vertreter des preußischen Gesundheitswesens eine Schlüsselrolle zu. Tatsächlich berichtete Kirchner auf der konstituierenden Sitzung, dass er Leydens Idee einer Krebssammelforschung sofort zugestimmt habe, nachdem ihm dieser im November 1899 diesen Plan auseinandergesetzt hatte. Als Hygieniker und ehemaliger Mitarbeiter Kochs konnte er, wie er selber wiedergab, der Argumentationskette gut folgen:

1. Es handelt sich hier um eine Krankheit, welche man lange Zeit als ein unvermeidliches Unglück angesehen hat. 2. Seit man jedoch eine parasitäre Ursache für dieselbe annimmt, welcher Ansicht auch der Redner zuneigt [hier Kirchner], ergibt sich auch die Möglichkeit, die Verhütung dieser Erkrankung ernstlich ins Auge zu fassen. 3. Da es die Aufgabe des Staates ist, sich an der Bekämpfung der vermeidbaren Krankheiten zu beteiligen, so kann es keinen Zweifel unterliegen, dass die Staatsbehörden sich betheiligen werden. (Comité für Krebsforschung 1902: 5)

Kirchner ist ein Beispiel par excellence für die Leyden'sche Ressourcenpolitik: Er gehörte zu jenen, die einen bakteriologischen Ansatz zur Klärung der Krebsätiologie favorisierten und hatte sich im Rahmen der Organisation der Tuberkulosebekämpfung engagiert. Darüber hinaus stellte er eine Verbindung zu den staatlichen Stellen her, die sowohl auf der politischen als auch der finanziellen Ebene eine Institutionalisierung stützen konnten.

Ebenso wie Kirchner verband Albert Guttstadt Person und Institution zum Vorteil der Institutionalisierung. Ab 1874 Dezernent für Medizinalstatistik beim Königlich Preussischen Statistischen Bureau verantwortete er die statistischen Daten, auf die sich Leydens Argumentation stützte. Die persönliche Anwesenheit Guttstadts und die von ihm vertretene Institution 


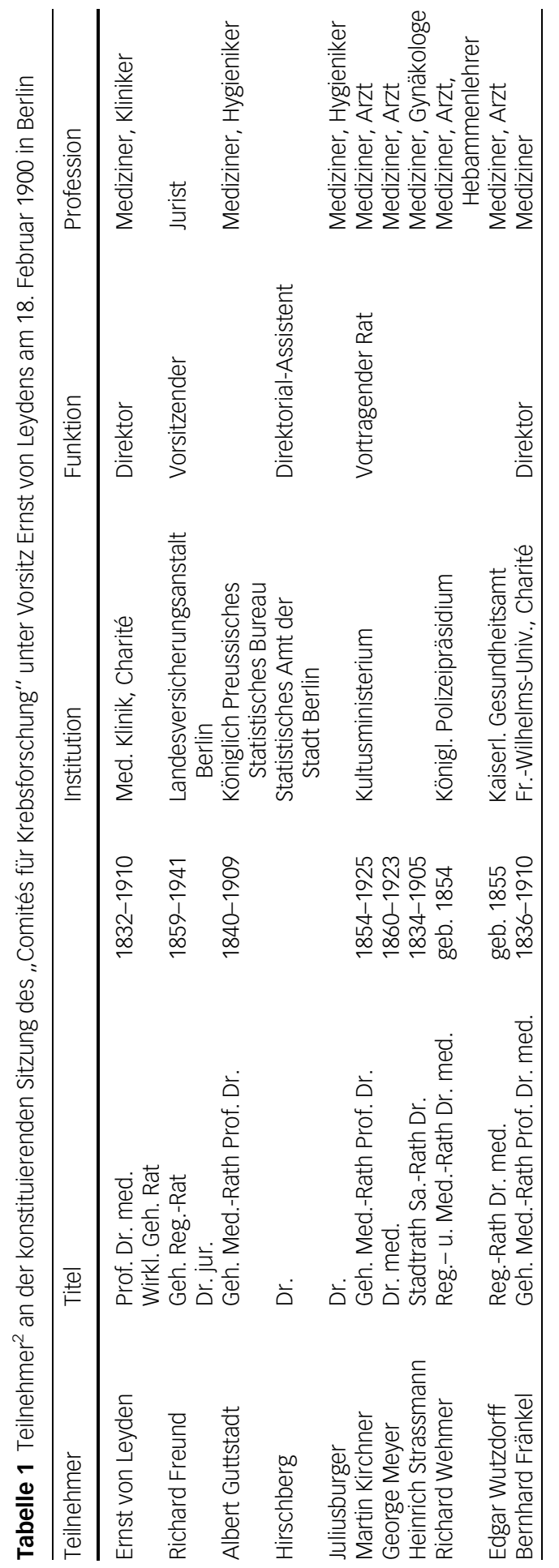


garantierten eine sichere Datenbasis. Das Organisationssystem „Statistisches Büro" war, wie Michael Schneider (2010) gezeigt hat, Mitte des 19. Jahrhunderts zunächst dem Funktionssystem „Politik“ zugeordnet, orientierte sich ab 1860 aber zunehmend am Funktionssystem „Wissenschaft“. Diese Entwicklung im Selbstverständnis des Statistischen Bureaus ist aufschlussreich für die Anwesenheit eines seiner Vertreter. War zunächst „die konzeptionelle Trennung der Datensammlung durch das Bureau einerseits und ihre Auswertung [durch Wissenschaftler] andererseits erkennbar" (ebd.: 272), wird nun der eigene wissenschaftliche Anspruch deutlich. Gerade die Verortung zwischen Wissenschaft und Politik machte es der Statistik möglich, im Rahmen der Institutionalisierung ihren Beitrag in Form einer wissenschaftspolitischen Ressource zu leisten. Für das „Bureau Guttstadt“ war eine Kooperation mit externen Kapazitäten auf wissenschaftlichem Gebiet bereits etabliert (Strassmann 2006: 57). Es war somit zusätzlich als Bindeglied zwischen Politik und Wissenschaft zu verstehen und ermöglichte darüber hinaus, wie im Fall von Kirchner, einen Zugriff auf weitere Ressourcen.

\section{Die stufenweise Einbindung weiterer Teilöffentlichkeiten}

Die These eines dynamischen, prozesshaften Vorgangs der Institutionalisierung beinhaltet, dass diese mit der Konstituierung des „Comités für Krebsforschung" am 18. Februar 1900 keineswegs abgeschlossen war und es anschließend zu einer Einbindung weiterer Teilöffentlichkeiten im Sinne Schirrmachers kam. Die Antwort auf die Frage nach der treibenden Kraft hinter dieser Entwicklung ergibt sich aus den Zielen der Institutionalisierung selbst, die Leyden in Bezug auf die Krebserkrankung so beschrieb:

Können wir hoffen, durch gemeinsame Arbeit ein Heilmittel zu finden? Ein solcher Anspruch ist von vornherein abzulehnen. Denn diese Aufgabe ist zur Zeit unlösbar. Aber was auch bei anderen Seuchen gelungen ist, durch genaue Kenntniss ihrer Verbreitung, ihrer Ursachen, ihrer Sterblichkeit eine gewisse Prophylaxe zu finden, das liegt in den Grenzen der Möglichkeit. (Comité für Krebsforschung 1902: 3)

In seinem Institutionalisierungsprogramm mobilisierte Leyden die Grundlagenwissenschaft, indem er die Klärung der Ätiologie forderte, und zugleich an das Nützlichkeitsdenken appellierte, indem er die Prophylaxe thematisierte. Er rief die Fachwissenschaft und Fachkreise zu Forschungsanstrengungen und die Öffentlichkeit zur Vorsorge auf. Obwohl unter Prophylaxe zunächst unter Bezugnahme auf die Infektionskrankheiten Maßnahmen im Rahmen der Gesundheitserziehung $\mathrm{zu}$ verstehen waren, ging es Leyden auch um eine Einrichtung von Heilstätten für Krebskranke am Vorbild der Tuberkulose-Heilstätten. Das Programm implizierte die beobachtete Dynamik. 
Bisher habe ich argumentiert, dass Leydens Publikationen zur Krebsätiologie wesentlich zu seiner Motivation beitrugen und als Teil der Geschichte der Institutionalisierung zu interpretieren sind. Nach der Konstituierung des „Comités“ wurden zunächst die vorhandenen Strukturen unter Einbeziehung assoziierter Fachkreise und der Fachöffentlichkeit ausgebaut: Der Mitgliederkreis wurde durch Zuwahlen erweitert und es wurden zwischen 1903 und 1914 elf Landeskomitees gegründet. „Die Einrichtung von Landeskomitees schien geboten, um die Arbeiten in möglichst kleinen Bezirken unter Berücksichtigung aller örtlichen Verhältnisse zu dezentralisieren." (Meyer 1911: 10) Angesprochen waren hier in erster Linie Ärzte, Kliniker, Krankenhäuser, Pflegeanstalten, aber auch Tierärzte (Beck 1905: 12-13), also Vertreter der Fachdisziplin.

Mit der ab 1904 erscheinenden „Zeitschrift für Krebsforschung“3 ${ }^{\text {"3 }}$ urde ein eigenes Publikationsorgan geschaffen. Auch hier wurden die Adressaten deutlich benannt: „Ärztliche Kreise“ und „Forscher“. Der Kreis der Fachwissenschaft, der durch die professionelle Zugehörigkeit der Mitglieder der konstituierenden Sitzung definiert wurde, erfuhr eine Erweiterung, indem Fachleute außerhalb des engeren Forschungsgebietes eingebunden wurden. Ging es im Kontext der konstituierenden Sitzung um Legitimation auf argumentativer Ebene unter Bezugnahme auf Statistik, Bakteriologie und Tuberkuloseforschung, die alle wissenschaftspolitisch mobilisiert wurden, ging es jetzt um die Verfügbarkeit personeller Ressourcen. Sie hatten eine Multiplikatorfunktion zu erfüllen, die es der Kerngruppe ermöglichte, sich reichsweit als Interessenvertreter der Krebsforschung zu etablieren.

Die Inhalte des Vermittlungsprozesses umfassten die Lehrmeinung der Fachwissenschaft, aber auch die Aufklärung über Krebs unter der breiten Öffentlichkeit:

Nach den vorliegenden Beobachtungen darf man aber schon jetzt die Krebsbekämpfung für eine der wichtigsten Aufgaben der öffentlichen Gesundheitspflege erachten. Hand in Hand mit ihr muß eine Bekämpfung der Kurpfuscherei und besonders der Naturheilvereine gehen, da diese durch ihre Behauptung, daß der Krebs ohne Operation heilbar sei, viele Kranke abhalten, sich rechtzeitig zur operativen Behandlung zu entschließen ... . (Komitee für Krebsforschung 1904: 12)

Jedoch war das Ziel der „Bekämpfung der Kurpfuscherei“ nur die halbe Wahrheit, denn auch die eigene Klientel galt für Leyden als Zielgruppe: „Dementsprechend sind auch die Aerzte, welche noch heute den Krebs für den Ausdruck einer Konstituierungskrankheit erachten, gegen die Operation desselben: sie sehen Metastasen als Ausdruck einer Konstitutionskrankheit an." (Leyden 1904: 306)

Überzeugungsarbeit musste somit zunächst auch innerhalb der Fachkreise geleistet werden, denn eine öffentliche Diskussion eines derart zentralen Themas konnte nicht im Interesse der Fachwissenschaft liegen. Hier ging es 
um die bisher kontrovers diskutierte Frage einer parasitären Ätiologie. Daher wurden auch zunehmend wissenschaftlich konkurrierende Anschauungen vorgetragen, was im deutlichen Kontrast zu den Diskussionen in der Konstituierungsphase stand. Diese Kontroversen hatten eine Beschränkung des Kommunikationsprozesses auf die Fachwissenschaft zur Folge und wirkten einer Kommunikation nach außen entgegen.

Gerade die 1903 in Dresden veranstaltete Ausstellung „Volkskrankheiten und ihre Bekämpfung“ hatte gezeigt, wie der Anspruch „naturwissenschaftliches-medizinisches Wissen in allgemeinverständlicher Form darzustellen“, umgesetzt werden sollte. Denn es ging in dieser „kleinen Schau“ nicht nur darum, Expertenwissen an das Laienpublikum zu vermitteln, sondern ,auch um eine Form der öffentlichen Zelebrierung der Bakteriologie, die im Interesse von Forschung, Ärzteschaft, Staat und Wirtschaft darauf ausgerichtet war, medizinischem Denken und daraus abgeleitetem Handeln Anerkennung und Akzeptanz zu verschaffen" (Brecht 1999: 54, siehe auch Brecht und Nikolow 2000).

Hieran anzuschließen war den Krebsforschern noch nahezu unmöglich. Auch die Forderung, dass die Krebsforschung unter „Heranziehung der therapeutischen Bestrebungen“ an die "Oeffentlichkeit treten kann und soll“, überzeugt nicht. Die Operation war nun mal das einzige Mittel, das die Medizin bisher anzubieten hatte. Noch 1905 schrieb Czerny, „durch Operationen wird etwa ein Viertel der frühzeitig zur Behandlung kommenden Kranken gerettet, während die anderen unerbittlich dem traurigen Lose verfallen" (zit. nach Lindner 2009: 214). Und die Betroffenen, die frühzeitig kamen, gehörten der kleineren Gruppe an. Die Strahlentherapie stand erst am Beginn ihrer Entwicklung, das galt für die Anwendung der ionisierenden Strahlung ebenso wie für theoretische Erörterungen in Bezug auf die Wirkungsmechanismen. Als „Spitzenmedizin“ war die Radiumtherapie kaum für jedermann zugänglich.

Dennoch verweist das Protokoll der Sitzung des Komitees für Krebsforschung am 30. Juni 1904 darauf, dass von „beachtungswerter Seite ein Wink dahin gegeben worden [ist], daß das Komitee für Krebsforschung anfangen möge, sich allmählich nach außen hin zu betätigen." (Komitee für Krebsforschung 1904: 62) Dieser „Wink“ deutet darauf hin, dass eine Kommunikation nach außen bis dahin tatsächlich noch nicht oder kaum stattgefunden hatte. Von wem der genannte Wink kam, verriet Leyden in der Sitzung nicht. Obwohl das Komitee zunächst nicht die Absicht hatte, sich sogleich in der Öffentlichkeit zu betätigen, hielt er den Zeitpunkt dennoch für sehr gut gewählt, an das „große Publikum“ heranzutreten, dessen größtes Interesse „die Frage der Prognose und der etwaigen Heilung des Krebses [ist]" (ebd.).

Aus der Diskussion um die Verfahrensweise in dieser Frage trat Leyden mit „von dem Komitee günstig aufgenommenen Vorschlägen“ hervor. Sie 
beinhalteten die Schaffung einer Fürsorgestelle und die Errichtung von „Asylen oder Heimstätten für Krebskranke“, beide orientiert an den Asylen für Tuberkulosekranke. Der Plan für die Fürsorgestellen schloss die Radiumbehandlung als einen wesentlichen Teil ein, ebenso die Behandlung mit Röntgenstrahlen. Die an Leydens Vortrag anschließende Diskussion griff lediglich die Frage nach der Errichtung von Asylen für Krebskranke auf. Der Schritt, auf das „große Publikum“ zuzugehen, unterblieb. War der Zeitpunkt hierzu nun doch nicht so „sehr gut gewählt“ wie Leyden vorgab?

Die Öffentlichkeit einzubeziehen, setzte eine erneute Rekonfiguration der Ressourcenensembles voraus. Hierfür waren Transaktionskosten zu leisten, die Leyden nicht bereit war, aufzubringen. Er konzentrierte sich in dieser Phase auf den innerwissenschaftlichen Diskurs. Mit der angeregten Diskussion um die Einrichtung von Fürsorgestellen und Asylen im Komitee wurde seinen Vorstellungen und Zielen entsprochen. Eine Beschränkung auf diese Fragestellungen, ohne eine Mobilisierung neuer Ressourcen, war für ihn letztendlich konsequent und zielgerichtet, wobei er den „Wink“ geschickt aufzunehmen verstand.

Eine Erweiterung des Adressatenkreises und die Einbindung weiterer Öffentlichkeiten konnten somit zu diesem Zeitpunkt nicht von Leyden ausgehen. Es war Vinzenz Czerny (1842-1916), der in Heidelberg für die Mobilisierung finanzieller Ressourcen die Bereitschaft aufbrachte, die notwendigen Transaktionskosten zu tragen.

Neben der Gründung von Landeskomitees und der Zeitschrift für Krebsforschung war die Einrichtung dedizierter Forschungsinstitute ein wesentlicher Schritt im Verlauf der Institutionalisierung der Krebsforschung. Die Gründungsgeschichten und Arbeits- bzw. Forschungsziele der Institute in Frankfurt am Main, Berlin und Heidelberg unterschieden sich teilweise deutlich voneinander und sind in der Medizingeschichte mehrfach diskutiert worden. Sowohl Paul Ehrlich in Frankfurt als auch Vinzenz Czerny in Heidelberg waren Mitglieder des Komitees für Krebsforschung, beide bereits in der ersten Mitgliederliste 1902 geführt. $^{4}$

In Berlin wurde schon am 8. Juni 1903 ein Krebsinstitut eröffnet. Die Mobilisierung politischer und finanzieller Ressourcen gelang Leyden im Rahmen des etablierten Netzwerks von Krebs- bzw. Tuberkuloseforschung und preußischer Administration. Leyden schrieb über diesen Vorgang, Friedrich Althoff selbst habe ihn gefragt, ob er nicht für seine Studien über den Krebs ein eigenes Institut haben wolle (Lohde-Boetticher 1910: 255). Die schnelle Institutsgründung in Berlin beruhte auf einer gegenseitigen Inanspruchnahme verschiedener Ressourcen: auf der einen Seite Geldmittel und politische Unterstützung zum Aufbau des Instituts, auf der anderen Seite eine Integration der Krebsforschung in die Berliner Wissenschaftslandschaft im Sinne der preußischen Wissenschaftsadministration um Friedrich Althoff. 
Gegenüber Frankfurt und Berlin, die der preußischen Wissenschaftsadministration unterstanden, verlief die Institutsgründung im badischen Heidelberg anders. Czerny fasste seine Gedanken „über die Einrichtung eines Instituts für experimentelle Krebsforschung erstmals 1901 in einem Aufsatz zusammen" (Lindner 2009: 200). Die Institutseröffnung erfolgte fünf Jahre später, im September 1906. Czerny benötigte deutlich länger als Leyden, um seine Ideen umzusetzen. Im Gegensatz zu Leydens Institut wurde das Heidelberger Institut erst durch Schenkungen und Spenden ermöglicht. Ein öffentlicher Aufruf 1905 zur Unterstützung führte Czerny zahlreiche Stifter und Wohltäter zu (ebd.: 214). Für ihn eröffnete eine deutlich offensivere Öffentlichkeitsarbeit erst die Chance, die Mittel bereitzustellen, die eine Institutsgründung möglich machten.

Leyden und Czerny unterschieden sich somit wesentlich in Bezug auf die zu mobilisierenden Ressourcen für die jeweiligen Institutsgründungen. Während sich Leyden weiterhin eines bewährten Ressourcenensembles bediente, bedurfte Czernys Vorgehensweise erst der Mobilisierung von Ressourcen durch die Öffentlichkeit, die es erforderte, den Aktionsraum zu erweitern. Der Paradigmenwechsel in Bezug auf die Öffentlichkeit war somit maßgeblich durch die Person Czernys bestimmt und nicht wissenschaftlicher Natur. So unterschiedlich die Gründungsmotive und - prozesse auch waren, die Einrichtung von Krebs(forschungs)instituten hatte letztlich dazu geführt, dass die Krebsthematik präsenter und insgesamt deutlicher wahrgenommen wurde. Es wurde eine Phase eingeleitet, in der die Krebsforschung begann, die Linie zu überschreiten, die den bisher betrachteten Raum der fachwissenschaftlich definierten „Teilöffentlichkeiten“ begrenzte. Die Institutsgründungen führten zu einer Wahrnehmung der Krankheit in der Öffentlichkeit, die auch mit Hoffnungen auf Heilung konnotiert war, womit Zukunftsperspektiven eröffnet wurden. Insbesondere Czerny, der im Gegensatz zu den preußischen Einrichtungen gezwungen war, für die Verwirklichung seines Heidelberger Instituts öffentlichkeitswirksam Mittel einzuwerben, transportierte Neugier, Interesse und auch Hoffnung in den öffentlichen Raum.

\section{Die Hinwendung zur breiten Öffentlichkeit}

Der erste Schritt in Richtung "großes Publikum“ erfolgte erst mit der Gründung der „Internationalen Vereinigung für Krebsforschung“ im Mai 1908. Hier wurde nach Korrektur des Satzungsentwurfs unter Name und Zweck $\$ 1$, 6. „die Verbreitung der Kenntnis über das Wesen der Krebskrankheit im Volke" aufgenommen (Internationale Vereinigung für Krebsforschung 1909: Anhang). Der notwendige Impuls kam hierbei von dem Vertreter ÖsterreichUngarns, Professor Julius von Hochenegg (1859-1940). Er schlug vor, „die 
Oeffentlichkeit mit der Krebsfrage mehr zu beschäftigen und auf diese Weise das Interesse für diese Krankheit und die Bereitwilligkeit zu finanzieller Unterstützung der Erforschung der Krankheit im Volke mehr zu fördern.“ (Ebd.: 11)

Der II. Internationale Chirurgenkongress in Brüssel vom 21. bis 25. September 1908 wurde auf Anregung von Professor Dollinger aus Budapest von einer Ausstellung, die die Krebsfrage behandelte, begleitet. An der Organisation beteiligten sich auch die deutschen Landeskomitees, deren Beitrag dort der umfangreichste war und entscheidend von Czerny geprägt wurde (ebd.: 90). Auch wurde beschlossen, dass im Interesse der Krebsforschung und der Volksbelehrung in jedem der zur Vereinigung gehörenden Länder ein Krebsmuseum gegründet werden solle (ebd.: 123). 1908 wurde die Zurückhaltung gegenüber dem Schritt in Richtung auf das große Publikum aufgegeben.

Über die Internationalisierung der Krebsforschung und die Verbindung von Fachkongress und Ausstellung gelang eine Einbindung der deutschen Wissenschaftler in das Ausstellungsgeschehen, bzw. in eine umfangreichere Öffentlichkeitsarbeit, die über das Verteilen von Broschüren und ähnliches hinausging. Auch die II. Internationale Konferenz für Krebsforschung im Oktober 1910 in Paris wurde von einer Ausstellung begleitet. Als Referat für die Konferenz wurde etwa die „Aufklärung des Publikums durch Vorträge und Ausstellungen" thematisiert (ebd.: 162).

Im Rahmen der Kongresse und Konferenzen wandte man sich zunächst an die Fachöffentlichkeit, bereitete aber auch die Einbindung der gebildeten und interessierten Öffentlichkeit vor. Für die Öffnung des Komitees für Krebsforschung gegenüber weiterer Teilöffentlichkeiten waren somit zwei Faktoren von Bedeutung: zum einen die Internationalisierung der Krebsforschung, zum zweiten die Person Czernys, der als Vertreter des Komitees die sich bietenden Möglichkeiten nutzte. ${ }^{5}$

Eine erste Anbindung an eine große, öffentliche Gesundheitsausstellung gelang mit der Internationalen Hygiene-Ausstellung 1911 in Dresden. Obwohl die "Gruppe Krebs“ eine der wissenschaftlichen Abteilungen war, stand sie doch jedermann offen, was im Rahmen der Fachkongresse und Fachausstellungen nicht gegeben war. 1911 gelang es durch die Integration wissenschaftlicher Abteilungen in die Gesamtausstellung, sowohl die Fachöffentlichkeit als auch die "gebildete und interessierte Öffentlichkeit" anzusprechen. Aber auch der "gelegentlich interessierten Öffentlichkeit", die das eigentliche Massenpublikum bildete, war der Zugang möglich.

Den Vorsitz der "Gruppe Krebs“ übernahm Czerny, als stellvertretende Vorsitzende wurden Dr. Georg Schmorl, Geheimer Medizinalrat und Prosektor am Stadtkrankenhaus Friedrichstadt in Dresden, sowie Dr. David von Hansemann, Professor, Geheimer Medizinalrat und Prosektor am RudolfVirchow-Krankenhaus in Berlin, bestimmt (Verlag der Internationalen 
Hygiene-Ausstellung Dresden 1911: 3). Die Hälfte der ca. 30 Aussteller kam aus dem Vorstand des Deutschen Zentralkomitees für Krebsforschung oder waren lokale Dresdner Ärzte. Czerny, Hansemann und Schmorl stellten etwa dreißig Prozent der Exponate, sie waren in Zusammenarbeit mit den lokalen Dresdner Kräften die zentralen Akteure.

Die Dominanz des Zentralkomitees bestätigt die Einbindung der Veranstaltung in den Institutionalisierungsprozess. Bereits 1906 hatte sich ein „Komitee für die in Dresden geplante Allgemeine Internationale HygieneAusstellung" konstituiert. Neben den Vertretern der medizinischen und hygienischen Fachbereiche waren auf politischer Ebene hohe Repräsentanten des Reichs, des Landes Baden und der Stadt Dresden zugegen. Karl August Lingner wurde mit der Planung und der geschäftlichen Leitung des Unternehmens beauftragt, später war er auch Vorsitzender des Direktoriums. Obwohl der Name Lingners im Allgemeinen direkt mit der Internationalen Hygiene-Ausstellung verknüpft wird, ist diese kaum nur auf seinen Namen zu reduzieren. Den Dresdner Initiatoren gelang es geschickt, reichs-, lokal- sowie wissenschaftspolitische Ressourcen $\mathrm{zu}$ mobilisieren und miteinander $\mathrm{zu}$ verknüpfen.

Die Einbeziehung von Martin Kirchner als Vertreter des preußischen Gesundheitswesens und von Franz Bumm, dem Präsidenten des Kaiserlichen Reichsgesundheitsamtes, macht die Bedeutung auf politischer Ebene deutlich. Bumm hatte den Arzt Friedrich August Weber, Kaiserlicher Regierungsrat und Mitglied des Gesundheitsamtes in Berlin, zum 1. Juli 1909 für die wissenschaftliche Leitung freigestellt, ${ }^{6}$ das Königlich Sächsische Kriegsministerium stellte den Stabsarzt Dr. Xylander frei und das Ministerium des Innern den Regierungsassessor Dr. Steinbach. Bumm schrieb in Hinblick auf den internationalen Charakters der geplanten Ausstellung,

[E]s würde daher dem Ausland gegenüber einen schlechten Eindruck machen, wenn die deutschen wissenschaftlichen Institute sich fernhielten. Unter den Leitern dieser Institute bestehe große Arbeitslust und Arbeitsfreude für die Beteiligung an der Dresdner Ausstellung, aber leider fehle es ihnen an den nötigen Mitteln, die auf jeden Fall bewilligt werden müßten, wenn die Institute Sehenswertes für die Ausstellung liefern sollen. ${ }^{7}$

Wie groß tatsächlich die „Arbeitslust und Arbeitsfreude“ unter den Krebsforschern war, ist fraglich - schon Leyden begründete 1906 seine Zurückhaltung mit nicht ausreichenden Mitteln. Viel mehr spricht dafür, dass der Beitrag für die Ausstellung hauptsächlich politisch motiviert war. Das „Deutsche Zentralkomitee für Krebsforschung“ wurde in die Pflicht genommen, im friedlichen „Kampf der Nationen“ um wissenschaftliche Erfolge einen Beitrag zu leisten. Nach zehnjährigem Bestehen des „Komitees für Krebsforschung" war es jetzt selbst zu einer wissenschaftspolitischen Ressource für die Reichsbehörden geworden. 
Die ersten Programmentwürfe von Lingner verweisen noch auf keine eigene Gruppe oder Abteilung „Krebs“. ${ }^{8}$ Auch nahmen Czerny und Hansemann 1906 nicht an der konstituierenden Sitzung in Dresden teil, ${ }^{9}$ auch wenn davon auszugehen ist, dass sie über die Planungen informiert waren. Die Organisatoren der Ausstellung machten umfangreich Werbung für ihr Vorhaben und verschickten für die konstituierende Sitzung eine Vielzahl von Einladungen. In einem frühen Katalogentwurf werden Czerny, Schmorl und Hansemann in der Liste „Personalien nach dem Stande von Ende September 1909“, „Krebskrankheiten“, geführt. ${ }^{10}$ Möglicherweise war zunächst gar nicht geplant, das Thema Krebs im Rahmen der Ausstellung in einer eigenen Sondergruppe zu behandeln. Im weiteren Verlauf der Planung konnten Czerny und Hansemann für den Vorsitz der „Gruppe Krebs“ gewonnen werden.

Deutlich ist die Koinzidenz in Bezug auf die oben beschriebene Öffnung gegenüber den Fachausstellungen 1908 und 1910. Die Protokolle des Zentralkomitees sowie die der Internationalen Vereinigung für Krebsforschung thematisierten die Ausstellung 1911 kaum, ihre zugrunde liegenden Konzepte und Ziele wurden hier nicht diskutiert. Das deutet auf eine geringe wissenschaftliche Relevanz hin und stärkt die These einer eher wissenschaftspolitischen Fürsprache. Auch ging es um Werbung für die Wissenschaft. Hansemann schrieb in der sozialhygienischen Korrespondenz Hygieia, die von dem Nachrichtenbüro der Ausstellung herausgegeben wurde: „Wenn die hier kurz skizzierten Ziele der Popularisation der Krebsforschung erreicht werden, so dürfte die Abteilung „Krebs“ auf der Hygiene-Ausstellung von größten Nutzen für die Menschheit sein und dadurch ihre Berechtigung voll und ganz dokumentiert haben." (Hansemann 1911) Über die Ziele im Einzelnen:

Diese Abteilung hat [...] einen doppelten Zweck: Der erste ist der, weitere Kreise für diese Krankheit zu interessieren und dadurch die Erforschung des Krebses zu fördern. [...] Wenn die Ausstellung [...] die Aufmerksamkeit wohlhabender Kreise soweit zu wecken imstande wäre, daß dadurch größere Mittel zur Erforschung des Krebses zur Verfügung gestellt werden könnten, so würde damit ... ein sehr wesentlicher Dienst geleistet sein. Der andere Zweck aber ist der, in weitesten Kreisen bekannt zu machen, daß der Krebs heilbar ist, wenn er frühzeitig erkannt und frühzeitig operiert wird. (Ebd.)

Hinsichtlich der medizinischen Möglichkeiten, der Therapie, aber auch der Ätiologiedebatte, hatte die Krebsforschung 1911 gegenüber 1904 keine neuen Perspektiven erschlossen. So beschränkte sich Hansemann auf eine Rhetorik, die schon 1905 von Czerny zur Mobilisierung finanzieller Ressourcen genutzt wurde, auf die Notwendigkeit einer frühzeitigen Operation bei der Diagnose Krebs hinzuweisen. Hansemann selbst benannte als ein Ziel die Werbung und gab damit die Adressaten vor. Mit dieser Form der „Popularisierung“"11 der Krebsforschung wurde die Institutionalisierung 1911 zum Abschluss gebracht. Zugrunde lag diesem letzten, abschließenden Schritt ein erneuter Wandel des 
Ressourcenensembles. Dessen wesentliches Merkmal war nun, dass das Komitee für Krebsforschung jetzt selbst als wissenschaftspolitische Ressource mobilisiert wurde.

\section{Schluss}

Die konstituierende Sitzung des „Comités für Krebsforschung“, die am Anfang der über 100-jährigen Geschichte der institutionalisierten Krebsforschung steht, hat mittlerweile einen fast mythischen Charakter:,Am Sonntag, dem 18. Februar 1900, kamen im Sitzungssaal des preußischen Kultusministeriums in Berlin von 12:15 bis 13:45 Uhr zehn namhafte Persönlichkeiten aus Wissenschaft und Politik zusammen" (Schneck and Peter 2000: 23). Dieses Narrativ ist fester Bestandteil der Geschichte der Krebsforschung und benennt bereits die sozialen Teilsysteme, Wissenschaft und Politik, deren Allianz eine Institutionalisierung der Krebsforschung ermöglichte. Genauere Untersuchungen erfolgten bisher nicht. Auch nach der zu leistenden Arbeit, die im Anschluss an die Konstituierung für den Fortgang der Institutionalisierung aufgebracht werden musste, wurde bisher nicht gefragt.

Das „Ressourcenmodell“ und das „Stufenmodell der Öffentlichkeit“ stellen geeignete Instrumente für eine derartige Analyse zur Verfügung. Mit Hilfe des Ressourcenmodells lassen sich die im Laufe der Institutionalisierung getroffenen Entscheidungen erklären, von der Auswahl der Mitglieder der konstituierenden Sitzung, bis zum Beitrag der Krebsforscher an der Dresdner Hygiene-Ausstellung. Die Erweiterung des Adressatenkreises erforderte eine kontinuierliche Bindung weiterer Ressourcen und deren Wandel. Das ist einerseits verständlich, da auch die Strukturen der neu erschlossenen Teilöffentlichkeiten einem Wandel unterlagen. Andererseits impliziert der Ansatz, „Wissenschaft und Politik als Ressourcen füreinander" zu verstehen, auch die Frage nach der Bidirektionalität der Ressourcenbeziehungen und deren Symmetrie. Die Institutionalisierung wurde von der Fachwissenschaft initiiert und die Mobilisierung der verschiedenen Ressourcen wurde somit notwendig.

Insbesondere der Anschluss an die Bakteriologie als „der vom Staat getragenen Wissenschaft" war ein wichtiger strategischer Faktor in diesem Prozess. Diese bewusste Orientierung an dem bestehenden System schloss aber bereits das Potenzial ein, selbst zu einer wissenschaftspolitischen Ressource zu werden. Wesentlich für den Verlauf war das Ausbleiben „medizinischer Innovationen“. Das zu Beginn der Institutionalisierung gemachte Angebot der Krebsforscher an die Politik bestand in einer „Programmatik“, einem „Wechsel auf die Zukunft“ (Ash 2001: 122), der letztlich in Dresden nun - politisch gewollt - einzulösen war. Insofern ist diese 
Entwicklung charakteristisch für die Krebsforschung. Die Klärung der Ätiologie oder eine Erweiterung der therapeutischen Möglichkeiten hätten möglicherweise zu einem anderen Verlauf geführt.

Zwischen 1906 und 1908 kam es zu einer stufenweisen Öffnung gegenüber der breiten Öffentlichkeit, die in der Programmatik der Institutionalisierung angelegt war, die aber erst extern durch die Internationalisierung der Krebsforschung und eine politische Mobilisierung durchgesetzt wurde. Schirrmacher verweist auf eine „signifikante Transformation im Verhältnis von Wissenschaft und Öffentlichkeit" in den Jahren um 1900, wobei er auf "Generationenwechsel, Mentalitätswandel und eine[r] Nachfrage nach der Verknüpfung von science et la vie“ (Schirrmacher 2008: 73/74) als Bestimmungsfaktoren dieser kulturellen Entwicklung hinweist. ${ }^{12}$

In Bezug auf die Wissenschaftsvermittlung bewegte sich die Krebsforschung somit grundsätzlich in einem Zeitfenster, in dem das Verhältnis zwischen Wissenschaft und Öffentlichkeit neu bestimmt wurde. Mit Hilfe des Konzepts der Ressourcenbeziehungen konnte diese Entwicklung konkretisiert werden. In Bezug auf wissenschaftliches Wissen wurde medizinisches Wissen von der Öffentlichkeit bevorzugt nachgefragt. Trotz einer Delegation an den Arzt waren Behandlungsmöglichkeiten und -folgen von besonderem Interesse (Schirrmacher 2008: 78).

Diese Themen wurden im Rahmen der Ausstellungen aufgegriffen, was auch Hansemann in den Zielen zum Ausdruck brachte. Die Pluralisierung der Öffentlichkeit in drei Schichten, wie von Schirrmacher vorgeschlagen, ließe sich am Beispiel der Krebsforschung genauer fassen. Eine Differenzierung zwischen „Patienten“, „Angehörigen“ und „allgemein Interessierten“ könnte eine Analyse der Pluralisierung von Vermittlungsdiskursen und Grenzziehungsarbeiten, wie von Schirrmacher angeführt (Schirrmacher 2008: 86), einleiten. Die organisierte Krebsforschung stand zwischen 1906 und 1908 am Beginn einer Entwicklung, in der die Wissensvermittlung in Bezug auf die Krebsthematik aufgriffen wurde, was heute ein fester Bestandteil der Wissensgesellschaft ist. Das vorgeschlagene Modell kann auch hier weitere Beiträge leisten.

\section{Danksagung}

Mein Dank gilt Herrn Professor Mikael Hård, Technische Universität Darmstadt, dessen Anregungen den Ausgangspunkt für die Beschäftigung mit dem Thema darstellte. Insbesondere möchte ich Frau PD. Dr. Sybilla Nikolow, Universität Bielefeld, danken. Ohne ihre stetige Diskussionsbereitschaft 


\section{Anmerkungen}

1 In den „Verhandlungen“ (Comité für Krebsforschung 1902: 1) heißt es: „Die Anwesenden konstituieren sich als Comité für Krebsforschung“. Ebert verweist auf das Protokoll der konstituierenden Sitzung (Ebert 1992: 128, Abb. 2), hier ist die Bezeichnung „Comité für Krebssammelforschung“. Auch Schneck (Schneck 2000: 23), sowie Atzl und Helms (2012: 14) nennen als Gründungsnamen "Comité für Krebssammelforschung“. Tatsächlich erfolgte bereits im Juni 1900 eine Umbenennung von „Comité für Krebssammelforschung" in „Komitee für Krebsforschung“. Da „Krebssammelforschung“ nur kurzzeitig Teil der Bezeichnung war, wurde im Rahmen dieses Aufsatzes durchgehend die Bezeichnung "Krebsforschung" gewählt, bzw. die in den „Verhandlungen“ gewählten Bezeichnungen zitiert.

2 Die biografischen Daten der Teilnehmer der konstituierenden Sitzung wurden folgenden Publikationen entnommen: Guttstadt, Historische Kommission 1966: 353; Hirschberg, keine Daten verfügbar; Juliusburger, keine Daten verfügbar; Kirchner, Killy/Vierhaus 1997: 553; Meyer, Degener 1909: 925; Strassmann, Strassmann 2006: Abb. 14, LangeQuassowski/Schneider 2012: 18; Wehmer, Degener 1909: 1515; Wutzdorf, Degener 1922: 1733; Fränkel, Killy 1996: 382.

3 Die Zeitschrift erscheint heute unter dem Titel: „Journal of Cancer Research and Clinical Oncology“.

4 Comité für Krebsforschung 1902, Mitgliederliste S. III-V. Czerny ist zwischen der 2. Sitzung, 21. März 1900, und der 3. Sitzung, 18. Juni 1900, beigetreten. Ehrlich wird auf der 9. Sitzung, 9. Juni 1902, als Mitglied genannt.

5 Gründe für Czernys Engagement wurden dargelegt. Der Einfluss, der von den Vertretern Österreich-Ungarns ausging kann hier nicht weiter diskutiert werden, ist aber sicherlich auch an den unterschiedlichen Forschermentalitäten der verschiedenen Länder festzumachen.

6 Bundesarchiv Berlin-Lichterfelde, R901/754.

7 Bundesarchiv Berlin-Lichterfelde, R901/753.

8 Hauptstaatarchiv Dresden, Bestand 10717, Signatur 8922, Blatt 69.

9 Ebd.

10 Ebd., Blatt 76.

11 Nach Schirrmacher sollte der Begriff der "Wissenschaftspopularisierung“ auf die zweite Hälfte des 19. Jahrhunderts beschränkt bleiben. Für die erste Hälfte des 20. Jahrhundert argumentiert Schirrmacher für die Verwendung des Begriffs der „Wissenschaftsvermittlung" (Schirrmacher 2008).

12 Schirrmacher bezieht sich hierbei auf die Printmedien. „Science et la vie“ war der Titel einer neuartigen populärwissenschaftlichen Zeitschrift, die ab 1913 erschien. Siehe hierzu Schirrmacher 2008, S. 74.

\section{Literatur}

Ash, Mitchell G., 2001. Wissenschaft und Politik als Ressourcen füreinander. Programmatische Überlegungen am Beispiel Deutschlands. In: Jürgen Büschenfeld, Heike Franz und FrankMichael Kuhlemann, Hg., Wissenschaftsgeschichte heute. Festschrift für Peter Lundgreen. Bielefeld, Gütersloh: Verlag für Regionalgeschichte, 117-134.

Ash, Mitchell G., 2007. Wissenschaft(en) und Öffentlichkeit(en) als Ressourcen füreinander. Weiterführende Bemerkungen zur Beziehungsgeschichte. In: Sybilla Nikolow und Arne 
Schirrmacher, Hg., Wissenschaft und Öffentlichkeit als Ressourcen füreinander. Frankfurt/ Main: Campus, 349-362.

Atzl, Isabel und Roland Helms, 2012. Die Geschichte der Deutschen Krebsgesellschaft. München: Zuckschwerdt.

Beck, Professor von, 1905. Die Gründung eines Landeskomitees in Baden. Zeitschrift für Krebsforschung (3): 12-13.

Berger, Silvia, 2009. Bakterien in Krieg und Frieden. Eine Geschichte der medizinischen Bakteriologie in Deutschland 1890-1933. Göttingen: Wallstein.

Brecht, Christine, 1999. Das Publikum belehren - Wissenschaft zelebrieren. Bakterien in der Ausstellung „Volkskrankheiten und ihre Bekämpfung“ von 1903. In: Christoph Gradmann und Thomas Schlich, Hg., Strategien der Kausalität. Konzepte der Krankheitsverursachung im 19. und 20. Jahrhundert. Pfaffenweiler: Centaurus, 53-76.

Brecht, Christine und Sybilla Nikolow, 2000. Displaying the Invisible: Volkskrankheiten on Exhibition in Imperial Germany. Studies in history and philosophy of biological and biomedical sciences (31): 511-530.

Comité für Krebsforschung, Hg., 1902. Verhandlungen des Comités für Krebsforschung. Heft I, 1900-1902. Berlin: Sonderdruck aus der Deutschen Medizinischen Wochenschrift.

Condrau, Flurin, 2000. Lungenheilanstalt und Patientenschicksal. Sozialgeschichte der Tuberkulose in Deutschland und England im späten 19. und frühen 20. Jahrhundert. Göttingen: Vandenhoeck \& Ruprecht.

Degener, Hermann A. L., Hg., 1909. Wer ist's. IV. Ausgabe. Leipzig: Verlag von H. A. Ludwig Degener.

Degener, Hermann A. L., Hg., 1922. Wer ist's. VIII. Ausgabe. Leipzig: Verlag von H. A. Ludwig Degener.

Ebert, Andreas, 1992. Das Protokollbuch I. Ein bemerkenswerter Fund zur Geschichte der Deutschen Krebsgesellschaft (1900-1907). Zeitschrift für die gesamte innere Medizin und ihre Grenzgebiete (47): 127-130.

Elkeles, Barbara, 1990. Der „Tuberkulinrausch“ von 1890. Deutsche medizinische Wochenschrift (115): 1729-1732.

Feinberg, Ludwig, 1907. Die Erreger und der Bau der Geschwülste. Insbesondere der Krebsgeschwülste. Berlin: R. Friedländer \& Sohn.

Gradmann, Christoph, 2010. Krankheit im Labor. Robert Koch und die medizinische Bakteriologie. Göttingen: Wallstein.

Hansemann, David von, 1911. Hygiene und Krebs. Hygieia, sozialhygienische Korrespondenz. Dresden: Herausgegeben von dem Nachrichten-Bureau der Internationalen HygieneAusstellung Dresden 1911 (28): ohne Seitenangabe.

Historische Kommission bei der Bayerischen Akademie der Wissenschaften, Hg., 1966. Neue Deutsche Biographie. 7. Bd. Berlin: Duncker \& Humblot.

Internationale Vereinigung für Krebsforschung, Hg., 1909. Cancer. Internationale Monatsschrift (1).

Killy, Walter, Hg., 1996. Deutsche Biographische Enzyklopädie. 3. Bd. Darmstadt: Wissenschaftliche Buchgesellschaft.

Killy, Walter und Rudolf Vierhaus, Hg., 1997. Deutsche Biographische Enzyklopädie. 5. Bd. Darmstadt: Wissenschaftliche Buchgesellschaft.

Komitee für Krebsforschung, Hg., 1904. Verhandlungen des Komitees für Krebsforschung. Heft III, 1903-1904. Berlin: Sonderdruck aus der Deutschen Medizinischen Wochenschrift.

Lange-Quassowski, Jutta und Volkmar Schneider, 2012. Eine bedeutende Ärztedynastie. Die Strassmanns. Berlin: Hentrich \& Hentrich.

Leyden, Ernst von, 1894. Ueber die Versorgung tuberkulöser Kranker seitens grosser Städte. Vortrag auf dem VIII. internationalen Congress für Hygiene zu Budapest gehalten in der allgemeinen Sitzung am 7. September 1894. Berlin: Verlag von August Hirschwald.

Leyden, Ernst von, 1901. Zur Aetiologie des Carcinoms. Zeitschrift für klinische Medizin (43): 110.

Leyden, Ernst von, 1903. Das Denken in der heutigen Medizin. Festrede, gehalten am Stiftungstage der Kaiser Wilhelms-Akademie für das militärärztliche Bildungswesen, 2. December 1902. Berlin: Otto Lange.

Leyden, Ernst von, 1904. Weitere Untersuchungen zur Frage der Krebsparasiten. Zeitschrift für Krebsforschung (1): 293-316. 
Leyden, Ernst von, 1907. Die internationale Vereinigung zur Bekämpfung der Krebskrankheit. Internationale Wochenschrift für Wissenschaft Kunst und Technik, 21. Dezember 1907, 11931200 .

Leyden, Ernst von und Fritz Schaudinn, 1896. Leydenia gemmipara Schaudinn, ein neuer, in der Ascites-Flüssigkeit des lebenden Menschen gefundener amoebenähnlicher Rhizopode. Sitzungsberichte der Berliner Akademie der Wissenschaften, 1896, 951-964.

Lindner, Cornelia, 2009. Vinzenz Czerny, Pionier der Chirurgie, chirurgischen Onkologie und integrierten Krebsforschung. Freiburg: Centaurus.

Lohde-Boetticher, Clarissa, Hg., 1910. Ernst von Leyden, Lebenserinnerungen. Stuttgart: Deutsche Verlagsanstalt.

Lüdtke, Karlheinz, 2002. Zur Entscheidbarkeit wissenschaftlicher Kontroversen [erörtert am Beispiel einer Auseinandersetzung in der früheren Geschwulstforschung]. Berlin: Max-PlanckInstitut für Wissenschaftsgeschichte (= Reprint 214).

Lüdtke, Karlheinz, 2007. Wissenschaftliche Kontroversen und die Entwicklung neuen Wissens in der Geschichte der Krebsforschung. Berlin: Gesellschaft für Wissenschaftsforschung.

Meyer, George, 1911. Bericht über die zehnjährige Wirksamkeit des Deutschen Zentralkomitees für Krebsforschung. Zeitschrift für Krebsforschung, 8-33.

Nikolow, Sybilla und Arne Schirrmacher, Hg., 2007. Wissenschaft und Öffentlichkeit als Ressourcen füreinander. Studien zur Wissenschaftsgeschichte im 20. Jahrhundert. Frankfurt am Main: Campus.

Schadewaldt, Hans, 1982. Zur Geschichte der Entdeckung des Tuberkelbazillus. Die medizinische Welt (33): 419-426.

Schirrmacher, Arne, 2008. Nach der Popularisierung. Zur Relation von Wissenschaft und Öffentlichkeit im 20. Jahrhundert. Geschichte und Gesellschaft (34): 73-95.

Schneck, Peter, 2000. Das Deutsche Zentralkomitee zur Erforschung und Bekämpfung der Krebskrankheit und seine Leistungen (1900-1933). In: Wolfgang Eckardt, Hg., 100 Jahre organisierte Krebsforschung. Heidelberg: Thieme, 23-29.

Schneider, Michael C., 2006 [recte: 2007]. Medizinalstatistik im Spannungsfeld divergierender Interessen. Kooperationsformen zwischen statistischen Ämtern und dem Kaiserlichen Gesundheitsamt/Reichsgesundheitsamt. In: Axel C. Hüntelmann, Johannes Vossen und Herwig Czech, Hg., Gesundheit und Staat. Studien zur Geschichte der Gesundheitsämter in Deutschland 1870-1950. Husum, 49-62.

Schneider, Michael C., 2010. Das Preußische Statistische Bureau in der zweiten Hälfte des 19. Jahrhunderts als Organisation der Wissensproduktion. In: Axel C. Hüntelmann und Michael C. Schneider, Hg., Jenseits von Humboldt. Wissenschaft im Staat 1850-1990. Frankfurt am Main: Peter Lang, Internationaler Verlag der Wissenschaften, 269-284 (= Berliner Beiträge zur Wissens- und Wissenschaftsgeschichte 13).

Strassmann, Wolfgang Paul, 2006. Die Strassmanns. Schicksale einer deutsch-jüdischen Familie über zwei Jahrhunderte. Frankfurt am Main: Campus.

Verlag der Internationalen Hygiene-Ausstellung, Hg., 1911. Sonder-Katalog der Gruppe Krebs der wissenschaftlichen Hygiene-Ausstellung Dresden 1911. Dresden: Verlag der Internationalen Hygiene-Ausstellung.

\section{Thorsten Kohl}

Technische Universität Darmstadt

Institut für Philosophie

Landwehrstraße 54

64293 Darmstadt

Germany

E-Mail: kohl@phil.tu-darmstadt.de 